\title{
CHARACTERIZATION OF APPROXIMATELY INNER AUTOMORPHISMS
}

\author{
MARIE CHODA
}

\begin{abstract}
Let $M$ be a finite factor acting standardly on a Hilbert space $H$. An automorphism $\theta$ of $M$ is approximately inner on $M$ if and only if there exists a state $\phi$ on $B(H)$ such that $\phi(J u J \theta(u))=1$ for every unitary $u$ in $M$, where $J$ is the canonical involution. Specially, $\theta$ is inner on $M$ if and only if such a state is a vector state.
\end{abstract}

1. Introduction. Let $M$ be a finite factor with the canonical trace $\tau$. An automorphism $\theta$ of $M$ is called approximately inner on $M$ if there is a sequence $\left(u_{n}\right)_{n}$ of unitaries in $M$ such that $\left\|\theta(x)-u_{n} x u_{n}^{*}\right\|_{2}$ converges to 0 as $n$ tends to infinity, for every $x$ in $M$. Let $H=L^{2}(M, \tau)$ be the Hilbert space of closed operators affiliated with $M$ whose domain is strongly dense with respect to $M$ due to Dixmier [4] and Segal [7] and let $M$ act on $H$ standardly. Denote by $\eta$ the canonical imbedding of $M$ into $H$ and by $J$ the canonical involution induced by $\eta: J \eta(x)=\eta\left(x^{*}\right)$ $(x \in M)$.

In [1, Theorem 1], we showed that, if an automorphism $\theta$ of $M$ is approximately inner on $M$, then there exists a state $\phi$ on $B(H)$ such that $\phi(J u J \theta(u))=1$ for every unitary $u$ in $M$. The referee of the paper [1] and Professor Sakai proposed the following problem to the author: Is the converse of [1, Theorem 1] true?

In this paper, we shall give an affirmative answer to the problem in Theorem 2 , and an analogous characterization to Theorem 1 of inner automorphisms on finite factors in Theorem 3.

The author would like to express her hearty thanks to Professor Sakai and the referee of the Proceedings for proposing the above problem to her, and to Professor Araki for indicating the simple proof of Lemma 1 below.

2. Results. Let $\operatorname{Tr}$ be the usual trace on $B(H)$, where $B(H)$ is the set of all bounded linear operators on $H$. We shall denote by $\|x\|_{\mathrm{HS}}$ and $\|x\|_{\mathrm{Tr}}$ the HilbertSchmidt and trace norm of $x$ in $B(H):\|x\|_{\mathrm{HS}}=\operatorname{Tr}\left(x^{*} x\right)^{1 / 2},\|x\|_{\operatorname{Tr}}=\operatorname{Tr}(|x|)$.

We need the following Lemma 1 in order to prove Theorem 2. The following simple proof of Lemma 1 is due to Professor Araki.

LEMMA 1. Let $b$ be a positive Hilbert-Schmidt class operator on $H$ and $u$ anitary operator on $H$. Then

$$
2\left\|u b^{2}-b^{2}\right\|_{\mathrm{Tr}} \geqslant\|u b-b\|_{\mathrm{HS}}^{2}
$$

Received by the editors February 6, 1981 and, in revised form, April 22, 1981.

1980 Mathematics Subject Classification. Primary 46L10.

Key words and phrases. Factor, automorphism, state. 
Proof.

$$
\begin{aligned}
\|u b-b\|_{\mathrm{HS}}^{2} & =\operatorname{Tr}\left((u b-b)^{*}(u b-b)\right)=\operatorname{Tr}\left(b^{2}-b u b-b u^{*} b+b^{2}\right) \\
& =\operatorname{Tr}\left(b(1-u) b+b\left(1-u^{*}\right) b\right) \\
& =2 \operatorname{Tr}(\operatorname{Re}(b(1-u) b))=2 \operatorname{Tr}(b(1-\operatorname{Re} u) b) \\
& \leqslant 2|\operatorname{Tr}(b(1-\operatorname{Re} u) b)-i \operatorname{Tr}(b(\operatorname{Im} u) b)| \\
& =2|\operatorname{Tr}(b(1-u) b)|=2\left|\operatorname{Tr}\left(b^{2}-u b^{2}\right)\right| \\
& \leqslant 2 \operatorname{Tr}\left(\left|b^{2}-u b^{2}\right|\right)=2\left\|b^{2}-u b^{2}\right\|_{\mathrm{Tr}}
\end{aligned}
$$

THEOREM 2. Let $M$ be a factor of type $\mathrm{II}_{1}$ with the separable predual (acting on $H=L^{2}(M, \tau)$ standardly for the canonical trace $\left.\tau\right)$. An automorphism $\theta$ of $M$ is approximately inner on $M$ if and only if there exists a state $\phi$ on $B(H)$ such that $\phi(J u J \theta(u))=1$ for every unitary $u$ in $M$.

Proof. Assume that, for an automorphism $\theta$ of $M$, there exists a state $\phi$ on $B(H)$ such that $\phi(J u J \theta(u))=1$ for every unitary $u$ in $M$. By [2, Theorem 3.1], It is sufficient to prove that, for any finite set $\left\{u_{1}, \ldots, u_{n}\right\}$ of unitaries in $M$ and any $\varepsilon>0$, there exists a $\xi$ in $H$ such that $\|\xi\|=1$ and $\left\|\xi-J u_{j} J \theta\left(u_{j}\right) \xi\right\|<\varepsilon$ for all $j=1, \ldots, n$. Take and fix a finite set $\left\{u_{1}, \ldots, u_{n}\right\}$ of unitaries in $M$ and $\varepsilon>0$. For each $j(1 \leqslant j \leqslant n)$, put $v_{j}=J u_{j} J \theta\left(u_{j}\right)$. Then $v_{j}$ is a unitary in $B(H)$. For each unitary $u$ in $B(H)$ and a state $\psi$ on $B(H)$, denote by $\psi u$ the linear functional such that $\psi u(x)=\psi(x u)$ for all $x$ in $B(H)$. For the predual $B(H)_{*}$ of $B(H)$, let $\left(B(H)_{*}\right)^{n}$ be the Banach space with norm $\left\|\left(\psi_{1}, \ldots, \psi_{n}\right)\right\|=\sum_{j=1}^{n}\left\|\psi_{j}\right\|$. Then $(B(H))^{n}$ is the dual of $\left(B(H)_{*}\right)^{n}$ by the duality

$$
\left(\phi_{1}, \ldots, \phi_{n}\right)\left(x_{1}, \ldots, x_{n}\right)=\sum_{j=1}^{n} \phi_{j}\left(x_{j}\right) \quad\left(\phi_{j} \in B(H)_{*}, x \in B(H)\right)
$$

Let $S$ be the set of all normal states on $B(H)$ and $W$ the set of all $\left(\psi-\psi v_{1}, \ldots, \psi\right.$ $\left.-\psi v_{n}\right)$, for $\psi$ in $S$. Since $S$ is weakly dense in the state space of $B(H)$, it follows that the state $\phi$ belongs to the weak closure of $S$. Hence $(0, \ldots, 0)=$ $\left(\phi-\phi v_{1}, \ldots, \phi-\phi v_{n}\right)$ belongs to the weak closure of $W$. On the other hand $W$ is a convex set. Therefore, the norm closure of $W$ contains $(0, \ldots, 0)$, so that we have a normal state $\psi$ on $B(H)$ such that $\sum_{j-1}^{n}\left\|\psi-\psi v_{j}\right\|<\varepsilon^{2} / 2$. Let $b$ be the positive Hilbert-Schmidt class operator on $H$ such that $\|b\|_{\mathrm{HS}}=1$ and that $\psi(x)=$ $(x b, b)_{\mathrm{HS}}$. Then, by Lemma 1 , we have that

$$
\varepsilon^{2}>2 \sum_{j=1}^{n}\left\|\psi-\psi v_{j}\right\|=2 \sum_{j=1}^{n}\left\|b^{2}-v_{j} b^{2}\right\|_{\mathrm{Tr}} \geqslant \sum_{j=1}^{n}\left\|b-v_{j} b\right\|_{\mathrm{HS}}^{2} .
$$

Let $b=\sum_{i=1}^{+\infty} \lambda_{i} \xi_{i} \otimes \xi_{i}$ be the form due to Schatten [6], then $\left(\lambda_{i}\right)_{i}$ is the set of eigenvalues of $b$ and $\left(\xi_{i}\right)_{i}$ is an orthonormal basis for $H$. 
Assume that there exists a $k(1 \leqslant k \leqslant n)$ such that $\left\|\xi_{i}-v_{k} \xi_{i}\right\| \geqslant \varepsilon$ for all $i$. Then we have that

$$
\begin{aligned}
\varepsilon^{2} & >\sum_{j=1}^{n}\left\|b-v_{j} b\right\|_{\mathrm{HS}}^{2}=\sum_{j=1}^{n} \operatorname{Tr}\left(\left(b-v_{j} b\right) *\left(b-v_{j} b\right)\right) \\
& =\sum_{j=1}^{n}\left(\sum_{i=1}^{+\infty}\left\|\left(b-v_{j} b\right) \xi_{i}\right\|^{2}\right)=\sum_{j}\left(\sum_{i}\left\|\lambda_{i}\left(\xi_{i}-v_{j} \xi_{i}\right)\right\|^{2}\right) \\
& =\sum_{j}\left(\sum_{i}\left|\lambda_{i}\right|^{2}\left\|\xi_{i}-v_{j} \xi_{i}\right\|^{2}\right) \\
& \geqslant \sum_{j \neq k}\left(\sum_{i}\left|\lambda_{i}\right|^{2}\left\|\xi_{i}-v_{j} \xi_{i}\right\|^{2}\right)+\sum_{i}\left|\lambda_{i}\right|^{2} \varepsilon^{2} \geqslant \varepsilon^{2},
\end{aligned}
$$

by the property that $\Sigma_{i}\left|\lambda_{i}\right|^{2}=\|b\|_{\mathrm{HS}}^{2}=1$. This is a contradiction. Hence we have a unit vector $\xi$ in $H$ such that $\left\|\xi-J u_{j} J \theta\left(u_{j}\right) \xi\right\|<\varepsilon$ for all $j(j=1, \ldots, n)$. Therefore, $\theta$ is approximately inner on $M$.

Conversely, if $\theta$ is approximately inner on $M$, by [1, Theorem 1], there exists a state $\phi$ on $B(H)$ with the desired property.

We shall call such a state $\phi$ as in Theorem 2 a state associated with $\theta$. Next, we shall show that the inner automorphisms of a finite factor $M$ are precisely those automorphisms, whose associated states are normal on $B(H)$. The following theorem is basically known in [3]. But we shall give a proof for completeness.

TheOREM 3. An automorphism $\theta$ of a finite factor $M$ (acting standardly on the Hilbert space $H=L^{2}(M, \tau)$ ) is inner if and only if there exists a unit vector $\xi$ in $H$ such that $(J u J \theta(u) \xi, \xi)=1$ for all unitary $u$ in $M$.

Proof. Let $\theta$ be an inner automorphism of $M$ and $v$ a unitary in $M$ inducing $\theta$. Put $\xi=\eta(v)$, then we have that $(J u J \theta(u) \eta(v), \eta(v))=\left(\eta\left(\theta(u) v u^{*}\right), \eta(v)\right)=\tau\left(v^{*} v\right)$ $=1$, for all unitaries $u$ in $M$.

Conversely, let $\xi$ be a vector in $H$ such that $\|\xi\|=1$ and $(J u J \theta(u) \xi, \xi)=1$ for all unitaries $u$ in $M$. Then we have that $\|J u J \theta(u) \xi-\xi\|^{2}=2\|\xi\|^{2}-(J u J \theta(u) \xi, \xi)-$ $(\xi, J u J \theta(u) \xi)=0$, so that $J u J \theta(u) \xi=\xi$ for all unitaries $u$ in $M$. Let $\xi=w|\xi|$ be the polar decomposition of the closed operator $\xi$, then $w$ is a partial isometry in $M$ and $|\xi|$ is a nonzero positive operator which is affiliated with $M$. Denote by $e_{t}$ the characteristic function of the real interval $(t,+\infty)$. Then there exists a positive real number $r$ such that $e_{r}(|\xi|) \neq 0$. Therefore, we have a nonzero operator $x=w e_{r}(|\xi|)$ in $M$ such that $\theta(u) x=x u$ for all unitaries $u$ in $M$, so that $\theta(x) a=a x$ for all $x$ in $M$. Since $M$ is factor, it follows that $\theta$ is inner on $M$.

The automorphism $\sigma$ on the tensor product $M \otimes M$ of a von Neumann algebra $M$ is called the symmetry of $M$ if it satisfies $\sigma(x \otimes y)=y \otimes x$ for all $x, y$ in $M$. In [5], Sakai proved that, if the symmetry of $M$ is inner on $M \otimes M$, then $M$ is type $I$. As an application of Theorem 3, we shall give another proof of this result for a finite factor $M$. Assume that the symmetry $\sigma$ of $M$ is inner on $M \otimes M$. Then by Theorem 3, we have a vector state $\phi$ on $B(H) \otimes B(H)$ such that

$$
\phi((J \otimes J) v(J \otimes J) \sigma(v))=1
$$


for all unitaries $v$ in $M \otimes M$. Put $\psi(x)=\phi(x \otimes 1)$ for all $x$ in $B(H)$. Then, by the proof of [1, Corollary 3], $\psi$ satisfies that $\psi(x a)=\psi(a x)$ for all $x$ in $B(H)$ and $a$ in $M$. On the other hand, $\phi\left(v^{\prime} \sigma(J \otimes J) v^{\prime}(J \otimes J)\right)=1$ for all unitaries $v^{\prime}$ in $M^{\prime} \otimes M^{\prime}$ because $J$ is a selfadjoint unitary and $J M J=M^{\prime}$. Hence for every unitary $u^{\prime}$ in $M^{\prime}$, $\phi\left(u^{\prime} \otimes J u^{\prime} J\right)=\phi\left(\left(u^{\prime} \otimes 1\right) \sigma\left(J u^{\prime} J \otimes 1\right)\right)=1$. Using Schwarz's inequality, we have that $\phi\left(\left(u^{\prime} \otimes J u^{\prime} J\right) z\right)=\phi\left(z\left(u^{\prime} \otimes J u^{\prime} J\right)\right)$ for all unitaries $u^{\prime}$ in $M^{\prime}$ and $z$ in $B(H) \otimes$ $B(H)$. Therefore, we have that

$$
\begin{aligned}
\psi\left(u^{\prime} x\right) & =\phi\left(u^{\prime} x \otimes 1\right)=\phi\left(\left(u^{\prime} \cap J u^{\prime} J\right)\left(x \otimes J u^{* *} J\right)\right) \\
& =\phi\left(\left(x \otimes J u^{\prime *} J\right)\left(u^{\prime} \otimes J u^{\prime} J\right)\right)=\psi\left(x u^{\prime}\right)
\end{aligned}
$$

for all unitaries $u^{\prime}$ in $M^{\prime}$ and $x$ in $B(H)$, so that $\psi\left(a^{\prime} x\right)=\psi\left(x a^{\prime}\right)$ for all $a^{\prime}$ in $M^{\prime}$ and $x$ in $B(H)$. Since $\phi$ is a vector state, it follows that $\psi$ is $\sigma$-weakly continuous on $B(H)$. So $\psi$ is a finite trace on $B(H)$, because $B(H)$ is generated by $M$ and $M^{\prime}$. Hence $H$ is finite-dimensional, and so $M$ is a finite factor of type $I$.

\section{REFERENCES}

1. M. Choda, The states associated with approximately inner automorphisms, Proc. Amer. Math. Soc. 81 (1981), 343-344.

2. A. Connes, Classification of injective factors, Ann. of Math. (2) 104 (1976), 73-115.

3. 151-153.

4. J. Dixmier, Formes linéaires sur un anneau d'opérateurs, Bull. Soc. Math. France 81 (1953), 9-39.

5. S. Sakai, Automorphisms and tensor products of operator algebras, Amer. J. Math. 97 (1975), 889-896.

6. R. Schatten, Norm ideals of completely continuous operators, Springer-Verlag, Berlin and New York, 1970.

7. I. Segal, A non-commutative extension of abstract integration, Ann. of Math. 57 (1953), 401-457.

Department of Mathematics, Osaka Kyoiku University, Tennoj, Osaka 543, Japan 